\title{
Meningoencephalitis due to Enteroviral Infection - An Often Overlooked Etiology
}

\author{
McBath A and Antony SJ
}

Texas Tech University School of Medicine, El Paso, Texas

${ }^{*}$ Corresponding author: Antony SJ, 1205 N Oregon, El Paso, Texas 79902, Fax: 915-533-4902, Tel: 15-5334900, E-mail: suresh.antony@att.net

Citation: McBath A, Antony SJ (2015) Meningoencephalitis due to Enteroviral Infection - An Often Overlooked Etiology. J Immunol Infect Dis 2(2): 203. doi: 10.15744/2394-6512.1.204

Received Date: December 14, 2014 Accepted Date: January 05, 2015 Published Date: January 09, 2015

\begin{abstract}
Enteroviruses are responsible for causing several recent well-publicized outbreaks in the United States, including gastrointestinal and upper respiratory infections. Enteroviruses are also capable of infecting the central nervous system, leading to manifestations of meningitis and encephalitis. Because enteroviruses are often overlooked as the etiology of CNS infections, we explore data from national surveillance, typical presentation and diagnostic information, as well as patterns of infection and transmission in an effort to remind practitioners to be mindful of this frequent cause of infection and its propensity to be spread from person to person. We present a case series that includes two confirmed cases of enteroviral meningoencephalitis, a presumed index case, and two cases involving children.
\end{abstract}

Keywords: Enterovirus; Encephailitis

\section{Introduction}

Enteroviruses have been implicated in recent outbreaks around the country, with symptoms ranging from gastrointestinal-like illness to respiratory infections. These viruses are well known causes of a much wider variety of clinical presentations and, due to the ease with which they spread from patient to patient, are a commonly reported cause of outbreaks. We report a case series of enteroviral infections of the central nervous system that involves three adults and two children, all of whom live in the same home.

Related to our case series, we also present some epidemiological and statistical data gathered from national enteroviral surveillance, including data from the Centers for Disease Control and Prevention. We also discuss several manifestations of enteroviral infection as well as clinical findings and diagnostic methods. Finally, because of its propensity to spread from person to person, we discuss transmission and reference published cases in which enteroviruses have been found to spread among patients in close contact, such as shared homes or classrooms.

\section{Case Reports}

\section{Index Case}

Our presumed index case is the husband of the patient presented in Case 1. We were unable to evaluate him directly because he was deployed overseas at the time of our first patient's presentation; thus our patient is the source of our data regarding this presumed index case. According to our patient, her husband began to feel sick and have headaches several days before being deployed, and he did not seek medical attention until he arrived at his destination. He was diagnosed with viral meningitis after a spinal tap was done. The exact viral etiology was not determined. According to the wife, he recovered after 72-96 hours and continued to stay well.

\section{Case 1}

Our first patient is a 30-year-old female who presented with headache, nausea, vomiting, and diarrhea. According to the patient, her symptoms began several days after her husband started showing symptoms. On admission her temperature was 98.2F, BP113/68 $\mathrm{mmHg}$, and pulse 78 beats per minute. On physical exam she was alert and oriented with mild nuchal rigidity; otherwise, the rest of the examination was unremarkable. The serum white blood count was 11,000 cells $/ \mathrm{mm}^{3}$, hemoglobin $13 \mathrm{~g} / \mathrm{dL}$, hematocrit $40 \%$ and platelets 301,000 cells $/ \mathrm{mm}^{3}$. Spinal fluid analysis revealed 8 leukocytes $/ \mathrm{mm}^{3}$ with $18 \%$ neutrophils and $81 \%$ lymphocytes, and glucose of $54 \mathrm{mg} / \mathrm{dL}$. CSF culture was negative, as were coagglutinins for Haemophilus influenza type B, Streptococcus pneumoniae, Group B streptococcus, and Neisseria meningitidis. HSV PCR was negative. Viral cultures were negative. MRI was unremarkable. A cerebrospinal fluid (CSF) sample sent for enteroviral RT- PCR analysis came back positive. Serotype was not done. She was treated with pain medications and intravenous fluids and recovered well. Follow up in 4 weeks was unremarkable. 


\section{Case 2}

This patient is the 29-year-old female roommate of the patient described above who presented with severe headaches around the same time the previously described patient had headaches. She also complained of a low grade fever and pain in the neck associated with photophobia and nausea but no vomiting.

Her temperature was 99 F, BP 123/64 mmHg, and pulse 72 beats per minute. Physical exam was significant for a young lady who looked uncomfortable due to neck pain, but she was alert and oriented. The rest of the physical examination was unremarkable. Her white blood cell count was 10,400 cells $/ \mathrm{mm}^{3}$, hemoglobin $11 \mathrm{~g} / \mathrm{dl}$, hematocrit $34 \%$, and platelets 298,000 cells/ mm 3 . CSF analysis showed 65 leukocytes $/ \mathrm{mm}^{3}$ with $87 \%$ lymphocytes and $7 \%$ neutrophils, and a protein of $63 \mathrm{mg} / \mathrm{dL}$. Viral cultures and HSV PCR were negative. Spinal fluid culture and coagglutinin assays were negative as above. Enteroviral RT-PCR came backpositive. She received similar treatment as the previous case and her recovery was unremarkable.

\section{Additional Cases}

In addition to the patients described above, two children (ages 5 and 7) in the household had similar symptoms (headaches, low grade fever, cough, and mild diarrhea) and were thought to have a viral illness. Many of their symptoms cleared up in 72 hours, but one of the children required admission for dehydration while the other child was treated at home.Both children were treated conservatively and did well (Table 1).

\begin{tabular}{|c|c|c|}
\hline Lab (units) & Patient 1 & Patient 2 \\
\hline \multicolumn{3}{|l|}{ Blood } \\
\hline Leukocytes $\left(1,000\right.$ cells $\left./ \mathrm{mm}^{3}\right)$ & 11 & 10.4 \\
\hline Hemoglobin (g/dL) & 13 & 11 \\
\hline Hematocrit (\%) & 40 & 34 \\
\hline Platelets $\left(1,000\right.$ cells $\left./ \mathrm{mm}^{3}\right)$ & 301 & 298 \\
\hline \multicolumn{3}{|l|}{ Cerebrospinal fluid } \\
\hline Red blood cells (cells $/ \mathrm{mm}^{3}$ ) & 0 & 0 \\
\hline Leukocytes (cells $/ \mathrm{mm}^{3}$ ) & 8 & 65 \\
\hline Neutrophils (\%) & 18 & 7 \\
\hline Lymphocytes (\%) & 81 & 87 \\
\hline Proteins (mg/dL) & 74 & 63 \\
\hline Glucose (mg/dL) & 54 & 48 \\
\hline Enteroviral PCR & positive & positive \\
\hline
\end{tabular}

\section{Discussion}

Table 1: Summary of blood and cerebrospinal fluid lab work from each patient

Enteroviruses are members of the family Picornaviridae and are responsible for a wide range of clinical manifestations, ranging from asymptomatic infection, mild febrile illness, conjunctivitis, and respiratory tract infections to more serious conditions such as meningoencephalitis and myocarditis [1-3]. The exact incidence of infection is difficult to appreciate due to the propensity of enteroviral infections to be asymptomatic [2], particularly in adults [4]. According to the CDC, an average of 1,467 cases per year were reported during surveillance from 1970-2005, with nearly half of infections being in children under one year old [5]. The true numbers, however, are certainly much higher because enteroviral infections are not nationally reportable; the CDC estimates that there are 10-15 million symptomatic enteroviral infections in the United States each year [5].

Infection patterns follow a temporal approach, with most enteroviral infections, including those responsible for meningitis, occurring during the summer months. According to national surveillance, states in the Southern United States have the highest rates of diagnosis [1]. Spread of infection may occur by either the fecal-oral or respiratory route [6]. In addition, the virus can be transmitted via fomites, facilitating transmission to those in close contact with infected individuals [7]. For this reason, transmission within households is relatively common [4]. In one study that examined household members of patients positively identified to have enteroviral infection, high rates of simultaneous infection were found in family members; for instance, there was an $84 \%$ transmission rate among siblings of infected individuals [4]. This same study also illustrates the increased likelihood of transmission between adults and children who are in close contact with each other: for children identified with enteroviral infection, $41 \%$ of parents were found to also be infected along with $28 \%$ of grandparents and $26 \%$ of aunts or uncles [4]. These findings are consistent with the cases described above with spread of infection between adults and children who share the same living space.

Enteroviruses are a common cause of meningitis, an infection of the protective membranes that surround the brain and spinal cord. Clinical manifestations usually have a sudden onset and are characterized by biphasic fever, headache, photophobia, vomiting, rash, and myalgia [3,6]. Infection may also spread to the brain parenchyma, a condition known as encephalitis, which may have focal neurologic signs or altered mental status in addition to the aforementioned symptoms. According to one 7-year observational study, enteroviruses were the most frequently identified viral cause of central nervous system infection in the cases analyzed [8]. 
While viral meningitis is often a benign and self-limited condition, physicians must be cautious in their evaluation in order to differentiate it from other causes such as bacterial meningitis, which is potentially life threatening if left untreated. The pattern of findings in the analysis of cerebrospinal fluid helps to differentiate the various etiologies of meningitis. In viral meningitis, CSF often shows a mild leukocytosis, usually with lymphocytic predominance; slightly elevated proteins; and normal glucose. In contrast, bacterial meningitis usually has markedly elevated leukocytes (hundreds to thousands per mm ${ }^{3}$ ) with neutrophilic predominance, elevated proteins, and low glucose [8]. While these guidelines are useful, actual samples from patients may deviate from these rules, so clinicians should use their best judgment when interpreting results of CSF analysis. For instance, early on in the course of viral meningitis, CSF often shows a neutrophilic predominance rather than the expected lymphocytic predominance, which may be misleading [9]. Differences in response to infection at various stages of life should also be taken into account during evaluation of suspected meningitis. For instance, it has been noted that children often have a greater systemic response to meningeal infection, showing higher blood leukocyte counts and fewer changes in CSF values as compared to adults [9]. Thus, the results of CSF analysis should be considered as part of a greater clinical picture when determining the etiology of meningeal infection.

An additional tool with great utility in the diagnosis of viral meningitis that has become widely available is polymerase chain reaction testing (PCR). Prior to PCR, viral meningitis was more or less a diagnosis of exclusion once a bacterial cause had been ruled out [6]. PCR is faster, more sensitive, and more reliable than viral culture in detecting enteroviruses [3]. Additionally, PCR is useful as a diagnostic tool because it can detect the presence of enteroviral RNA in cerebrospinal fluid that may have had leukocyte counts, protein levels, and glucose levels within normal limits [3]. The widespread availability of PCR has shown to save both time and money in the management of viral meningitis. PCR results can be obtained the same day the CSF is collected, whereas a bacterial culture may take several days and a viral culture even longer [10]. Patients who are rapidly diagnosed as having viral meningitis have also been shown to have shorter hospital stays and shorter duration of empiric treatment with antibiotics and/or acyclovir $[8,10]$.

PCR is not without its limitations, however. First, enteroviruses are present in the CSF only transiently; thus, they can only be detected for a short time [2]. Additionally, if the infection involves only the brain parenchyma (encephalitis) and not the meninges, PCR may fail [2]. Next, the utility of PCR testing in diagnosis of viral meningitis may be affected by temporal factors. One study found that the diagnostic yield of PCR in viral meningitis dropped if the fluid was collected more than two days after the onset of symptoms. The same study also found that using stool as the source for PCR provided a higher diagnostic yield after two days and up to weeks after symptom onset. However, because enteroviruses are a common cause of asymptomatic infections [11], detection of enteroviral RNA outside the cerebrospinal fluid could represent an incidental finding not necessarily related to the patient's clinical symptoms [11].

In conclusion, the case series we report here involving three adults and two children is consistent with current evidence that enteroviruses are capable of causing meningoencephalitis that is easily transmitted among close contacts. We use the presentation of the patients shown here with a review of the literature to remind clinicians of the role that enteroviruses play in various infections, their presentation and clinical findings, and that they should be kept in mind as a potential source of outbreaks.

\section{References}

1. Villarruel G, Langley G, Oberste M, Pallansch M (2010) Nonpolio enterovirus and human parechovirus surveillance -- United States, 2006-2008. MMWR Morb Mortal Wkly Rep 59: 1577-80.

2. Fowlkes A, Honarmand S, Glaser C, Yagi S, Schnurr D, et al. (2008) Enterovirus-associated encephalitis in the California encephalitis project, 1998-2005. J Infect Dis 198: 1685-91.

3. Tavakoli N, Wang H, Nattanmai S, Dupuis M, Fusco H, et al. (2008) Detection and typing of enteroviruses from CSF specimens from patients diagnosed with meningitis/encephalitis. J Clin Virol 43: 207-11.

4. Chang LY, Tsao KC, Hsia SH, Shih SR, Huang CG, et al. (2004) Transmission and clinical features of enterovirus 71 infections in household contacts in Taiwan. JAMA 291: 222-7.

5. Khetsuriani N, Lamonte-Fowlkes A, Oberste M, Pallansch M (2006) Enterovirus surveillance--United States, 1970-2005. MMWR Surveill Summ 55: 1-20.

6. Johns N (2012) Viral meningitis, active and reserve components, U.S. Armed Forces, 2002-2011. MSMR 19: 2-6.

7. Begier EM, Oberste MS, Landry ML, Brennan T, Mlynarski D, et al. (2008) An outbreak of concurrent echovirus 30 and coxsackievirus A1 infections associated with sea swimming among a group of travelers to Mexico. Clin Infect Dis 47: 616-23.

8. Big C, Reineck LA, Aronoff DM (2009) Viral infections of the central nervous system: a case-based review. Clin Med Res 7: 142-6.

9. Jimenez Caballero PE, Munoz Escudero F, Murcia Carretero S, Verdu Perez A (2011) Descriptive analysis of viral meningitis in a general hospital: differences in the characteristics between children and adults. Neurologia 26: 468-73.

10. Archimbaud C, Chambon M, Bailly JL, Petit I, Henquell C, et al. (2009) Impact of rapid enterovirus molecular diagnosis on the management of infants, children, and adults with aseptic meningitis. J Med Virol 81: 42-8.

11. Kupila L, Vuorinen T,Vainionpaa R, Marttila RJ, Kotilainen P (2005) Diagnosis of enteroviral meningitis by use of polymerase chain reaction of cerebrospinal fluid, stool, and serum specimens. Clin Infect Dis 40: 982-7. 


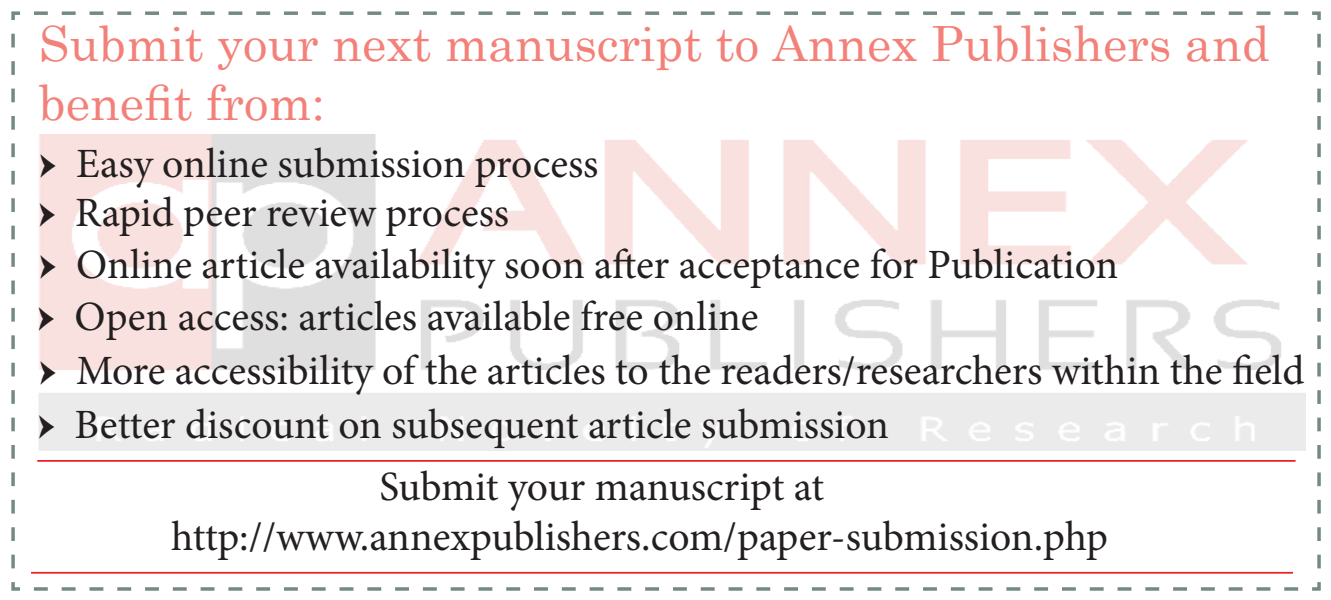

